\title{
A Nucleation Algorithm for the Coupled Conserved-Nonconserved Phase Field Model
}

\author{
A. M. Jokisaari ${ }^{a}$, C. Permann ${ }^{b}$, K. Thornton ${ }^{a}$ \\ ${ }^{a}$ Department of Materials Science and Engineering, College of Engineering, University of Michigan, Ann Arbor, MI \\ 48109, USA. ${ }^{b}$ Modeling and Simulation Department, Idaho National Laboratory, Idaho Falls, ID 83415, USA.
}

\begin{abstract}
This paper presents a refinement to the existing nucleation algorithm for a coupled conserved-nonconserved phase field model. In the new method, which offers greater ease of implementation as compared to the existing approach, only the nonconserved order parameter is modified to seed supercritical nuclei (thus termed order-parameter-only seeding). The order-parameter-only seeding method naturally satisfies the conservation law for the conserved order parameter. In addition, the implementation within a finite element framework is described. The evolution of a single nucleus is examined to ensure that the precipitate growth kinetics are not affected by the seeding method. We find that, after a brief initial transient period, order-parameter-only nucleation yields similar precipitate growth characteristics to that of the existing model. The kinetics of a phase transformation exhibiting concurrent nucleation and growth is analyzed in the form of the Avrami equation, and a statistical analysis is performed to determine if mesh and/or time adaptivity affects the simulation results. The statistical analysis indicates that the nucleation algorithm is amenable to adaptive meshing and adaptive time stepping.
\end{abstract}

keywords: phase field modeling, finite element method, nucleation, time adaptivity, mesh adaptivity.

\section{Introduction}

Nucleation and growth are key phenomena in phase transformations such as solidification and solid-solid precipitation. Nucleation behavior can have a major impact on the 
resulting microstructure and material properties. For example, precipitation hardening of alloys involves the formation of second-phase particles. The mechanical properties of such materials are dependent on precipitate size and spatial distribution; these characteristics are controlled in part by the nucleation behavior [1]. Thus, modeling of nucleation and growth is of major technological and scientific importance for materials development and design [2,3]. One means of modeling microstructural evolution is the phase field approach, which has been successfully employed to simulate phase transformations such as spinodal decomposition [4--7], coarsening [8--12], solidification [13--15], and thin film growth [6, 16--19]. Comprehensive descriptions and reviews of phase field modeling are found in Refs. [20--24].

In a phase field model, the microstructure is described by one or more continuous conserved or nonconserved field variables, termed order parameters. An order parameter is generally denoted as $\phi(\mathbf{r}, t)$ and indicates the phase at $\mathbf{r}$, where $\mathbf{r}$ is position and $t$ is time. Each phase is designated by a bulk value (e.g., $\phi=1$ for the $\alpha$ phase and $\phi=0$ for the $\beta$ phase), and the value of $\phi$ changes smoothly between phases to yield a diffuse interface that has a finite width. The position of the interface between the phases is described by an intermediate value (e.g., $\phi=0.5$ ). Thus, the phase field methodology eliminates the need to track interface positions explicitly. The free energy of the system is described as a functional of the order parameters, and the evolution of the system is driven by the reduction of the free energy.

Nucleation is commonly handled in one of two approaches within a phase field model [20], though other approaches exist as well. In the first method, a random noise term satisfying the fluctuation-dissipation theorem is added to the time evolution equation to model atomic-scale thermal fluctuations, giving rise to homogeneous nucleation [25]. However, the spatial and temporal resolutions required to accurately describe these fluctuations are computationally prohibitive with existing resources except for when modeling extremely small volumes. In practice, unphysically large random noise is often 
used in the early stage of a simulation to induce the formation of second-phase particles. After their formation, the noise is deactivated and growth of the particles ensues [25-27]. Similarly, heterogeneous nucleation on microstructural defects and walls has been modeled by the use of white- and colored-noise terms in the evolution equation [28].

An alternative to the aforementioned noise-based methods is explicit introduction of nuclei into the phase field simulation. In this method, classical nucleation theory is applied to a phase field model described by coupled conserved-nonconserved order parameters [29]. The local nucleation probability for each discretized volume in the simulation is calculated as a function of the local nucleation rate, and nucleation occurs stochastically as a function of the local nucleation probability. If nucleation occurs, a supercritical nucleus is introduced into the simulation by changing the value of the local composition field. To satisfy mass conservation, a depletion region around the nucleus must be created in the composition field. The algorithm was initially implemented with a depletion region described by a discontinuous concentration profile [29]. In subsequent work, a depletion region profile derived from the Zener gradient approximation [30] was demonstrated. This method was further modified by introducing a diffusion smoothing step after nucleus introduction [31] to create a smooth composition gradient at the nucleus/matrix interface.

Several other phase field treatments of nucleation exist in addition to the two aforementioned approaches. Microscopic phase field formulations of heterogeneous nucleation on defects were developed, in which the energy of a defect, such as a dislocation, is added into the local free energy to induce nucleation on the defect $[32,33]$. In addition, several methods have been developed to numerically determine the critical nucleus shape. In one approach, the critical nucleus profile under solidification conditions was determined by finding the time-independent solutions of the phase field evolution equations for a given concentration [34]. To obtain the shapes of critical nuclei in solid-solid phase transformations, which could deviate from a spherical shape due to elastic effects, both the nudged elastic band method [35] and the minimax technique [36--38] have been utilized. 
In this paper, a refinement to the existing nucleation algorithm for a coupled conservednonconserved phase field model is presented. In the new method, only the nonconserved order parameter is modified to seed supercritical nuclei (thus termed order-parameteronly, or OPO, seeding). The OPO seeding method is easier to implement than the original method $[29,30,39]$ and naturally satisfies the conservation law for the conserved order parameter. The implementation within a finite element framework is described, and the evolution of a single nucleus is discussed. The kinetics of a phase transformation exhibiting concurrent nucleation and growth is analyzed in the form of the Avrami equation, and a statistical analysis is performed to determine if mesh and/or time adaptivity affects the simulation results. While the implementation was performed within a finite element framework, the method may be used with other numerical methods such as the finite difference method.

\section{Methods}

\subsection{Phase field model}

The proposed OPO seeding method relies on the coupling between a conserved order parameter and a nonconserved order parameter through the free energy. Although OPO seeding can be implemented into any coupled conserved-nonconserved phase field model, a model describing the zirconium/zirconium hydride system [40] is adopted to demonstrate the approach. The zirconium/zirconium hydride system is described using a conserved field variable, $c$, that represents the concentration of hydrogen, and a nonconserved structural order parameter, $\eta$, that distinguishes the different structures of the two phases, where $\eta=0$ for zirconium and $\eta=1.5$ for the hydride. To demonstrate the nucleation algorithm, the free energy functional from Ref. [40] is simplified by neglecting elastic energy and by retaining only one structural order parameter corresponding to one 
of the three equivalent orientation variants. The free energy is given as

$$
F=\int_{V}\left\{f_{\text {chem }}(c, \eta)+\frac{\kappa_{c}}{2}|\nabla c|^{2}+\frac{\kappa_{\eta}}{2}|\nabla \eta|^{2}\right\} d V,
$$

where $\kappa_{c}$ is the gradient energy coefficient for concentration, $\kappa_{\eta}$ is the gradient energy coefficient for the structural order parameter, $f_{\text {chem }}$ is the bulk chemical free energy density, and $V$ is the volume. The expression for $f_{\text {chem }}$ used in this work is

$$
f_{\text {chem }}(c, \eta)=\frac{A_{1}}{2}\left(c-c_{1}\right)^{2}+\frac{A_{2}}{2}\left(c-c_{2}\right) \eta^{2}-\frac{A_{3}}{4} \eta^{4}+\frac{A_{4}}{6} \eta^{6},
$$

where $c_{1}, c_{2}, A_{i}(i=1, \ldots, 4)$ are constants. The values of $c_{1}$ and $c_{2}$ are the equilibrium concentrations of hydrogen in the matrix and hydride, respectively, while the $A_{i}$ 's control the shape of the free energy surface [40].

The microstructural evolution of the system is governed by coupled conserved-nonconserved dynamics. The Cahn-Hilliard equation [7] governs the evolution of the concentration of hydrogen as

$$
\frac{\partial c}{\partial t}=\nabla \cdot[M \nabla \mu]
$$

where the chemical potential, $\mu$, is

$$
\mu=\frac{\partial f_{\text {chem }}}{\partial c}-\kappa_{c} \nabla^{2} c
$$

$t$ is the time, and $M$ is the mobility of hydrogen. In addition, the Allen-Cahn equation [41] governs the evolution of the nonconserved structural order parameter as

$$
\frac{\partial \eta}{\partial t}=-L\left[\frac{\partial f_{\text {chem }}}{\partial \eta}-\kappa_{\eta} \nabla^{2} \eta\right]
$$

where $L$ is the kinetic coefficient. Both the mobility and the kinetic coefficient are assumed to be isotropic. Following Ref. [40], we use $L=M=0.4, A_{1}=18.5, A_{2}=-8.5$, 
$A_{3}=11.5, A_{4}=4.5, \kappa_{c}=\kappa_{\eta}=1.5, c_{1}=0.006$, and $c_{2}=0.59$ in nondimensionalized units. Nondimensionalization of the governing equations is found in the Appendix.

\subsection{Explicit nucleation algorithm}

Simmons et al. pioneered the development of an algorithm that explicitly introduces nuclei in a phase field simulation based on classical nucleation theory [29]. Hereafter, this method will be referred to as the explicit nucleation algorithm. The concepts from that algorithm that are incorporated into our model are summarized in this section. The local nucleation rate for critical nuclei, $J^{*}(\mathbf{r}, t)$, is calculated following classical nucleation theory [42] as [29]

$$
J^{*}(\mathbf{r}, t)=Z N \beta^{*} \exp \left(\frac{-\Delta G^{*}}{k_{B} T}\right) \exp \left(\frac{-\tau}{t}\right),
$$

where $Z$ is the Zeldovich correction factor, $N$ is the number of atoms in the element, $\beta^{*}$ is the frequency at which a critical nucleus becomes supercritical, $T$ is the temperature, $k_{B}$ is the Boltzmann constant, $\tau$ is the incubation time, and $\Delta G^{*}$ is the critical nucleus activation energy. For a given volume, the nucleation rate is then given by

$$
J^{*}(\mathbf{r}, t)=Z n \Delta v \beta^{*} \exp \left(\frac{-\Delta G^{*}}{k_{B} T}\right) \exp \left(\frac{-\tau}{t}\right),
$$

where $N=n \Delta v, n$ is the number density of atoms and $\Delta v$ is the volume of the element. Following Ref. [29], Eq. 6 is simplified to

$$
J^{*}(\mathbf{r}, t)=k_{1} \exp \left(\frac{-k_{2}}{\Delta c}\right)
$$

where $k_{1}$ and $k_{2}$ are adjustable parameters that control the nucleation rate and $\Delta c$ is the supersaturation. The local nucleation probability, $P(\mathbf{r}, t)$, is calculated as [29] 


$$
P(\mathbf{r}, t)=1-\exp \left(-J^{*} \Delta t\right),
$$

where $\Delta t$ is the time interval over which the nucleation probability is calculated.

In the explicit nucleation algorithm, the nucleation step is executed after each phase field time step [29], and $J^{*}$ and $P$ are calculated for each volume element in the simulation. It is assumed that nucleation rates are low enough for the given mesh that only one nucleus may form in any volume element [29]. After $P$ is computed, the positions of new nuclei are determined. This is accomplished by performing a binary test on the local nucleation probability: a random number, $R(t)$ between $[0,1]$ is generated, and if $R(t)<P(\mathbf{r}, t)$, a nucleus is introduced at that position [30].

\subsection{Order-parameter-only nucleation algorithm and implementation details}

The nucleation algorithm has been implemented in Hyrax, a finite element phase field simulation code. Hyrax is an application of the Multiphysics Object Oriented Simulation Environment (MOOSE) [43, 44], an open-source finite element partial differential equation framework. Hyrax is available online at http://gitub.com/UMThorntonGroup/hyrax, and MOOSE is found online at http://gitub.com/idaholab/moose, with additional documentation at http://mooseframework. org. MOOSE supports serial or parallel computation with adaptive time stepping and meshing, which can significantly reduce the computational resources required for large simulations. Within Hyrax, the discretizations of the weak forms of the Cahn-Hilliard and Allen-Cahn equations [45] are implemented using the "phase field" module within MOOSE. The reduced-order split Cahn-Hilliard formulation is used, in which an additional nonlinear variable is introduced to compute $\mu$ [46]. This approach offers significantly higher computational efficiency by allowing the use of lower order elements as compared to the full fourth-order Cahn-Hilliard formulation [46].

The implementation flow chart of the nucleation algorithm within Hyrax is shown in 
Fig. 1. After each phase field step, the nucleation step is executed. Within the nucleation step, $\Delta G^{*}, J^{*}$, and $P$ are calculated for each element (for the specific example in this paper, $\Delta c$ is calculated during the $\Delta G^{*}$ step). If the element contains a second-phase particle or is within the diffuse interface, the probability is set to zero; this is implemented by setting $P=0$ for $\eta>0.1$, although a different threshold value could be taken. After $P$ is computed, the positions of new nuclei are determined. In a similar manner as Ref. [30], a pseudorandom number, $R(\mathbf{r}, t)$, is generated with a uniform distribution between $[0,1]$ for each element. If $R(\mathbf{r}, t)<P(\mathbf{r}, t)$, a nucleus is introduced at that position. In the rare event that nucleation occurs in two adjacent elements, the two nuclei will merge into one precipitate. When a nucleation event occurs in a particular mesh element, the centroid of that element is chosen as the center point of the nucleus. The position of the nucleus and the time of its formation are stored in a list, which is accessed by the mesh adaptivity and nucleus introduction functions. If mesh adaptivity is enabled, the finite element mesh is refined as necessary so that the mesh has appropriate local resolution for the subsequent introduction of each nucleus. Nuclei are seeded accordingly by direct modification of the nodal solution vector of the structural order parameter field. If time adaptivity is used, the time step is reduced upon a nucleation event to a specified time step size. After the modification of the structural order parameter field, the system is allowed to evolve according to Eqs. 3 and 5.

To use the explicit nucleation algorithm with a nonuniform mesh, we define a volumetric nucleation rate, $\tilde{J}^{*}$. In this paper, $\tilde{J}^{*}$ is based on Eq. 8 and is formulated as

$$
\tilde{J}^{*}(\mathbf{r}, t)=\tilde{k}_{1} \cdot \exp \left(\frac{-k_{2}}{\Delta c}\right)
$$

where $\tilde{k}_{1}=k_{1} / \Delta v$. Thus, the nucleation probability for an element is given by

$$
P(\mathbf{r}, t)=1-\exp \left(-\tilde{J}^{*} \Delta v \Delta t\right)
$$




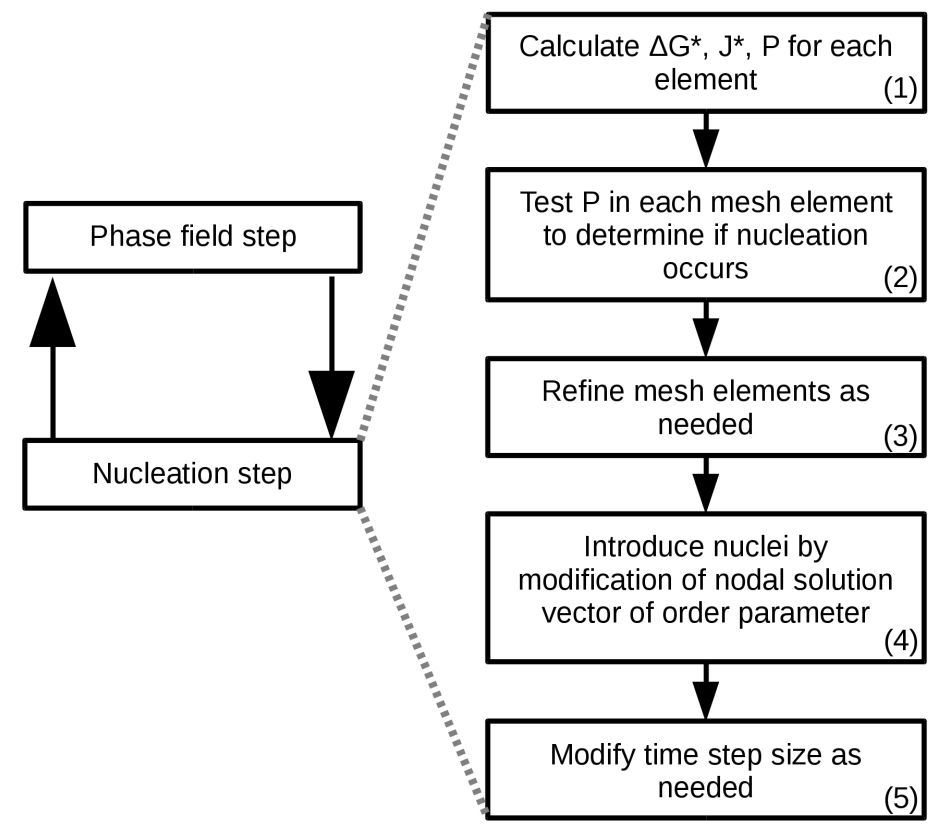

Figure 1: A flow chart of the order-parameter-only nucleation algorithm implemented within Hyrax. After a phase field time step, the nucleation algorithm is executed. (1) The nucleation activation energy, nucleation rate, and nucleation probability are calculated for each element. (2) The nucleation probability in each element is tested to determine if nucleation occurs. If mesh adaptivity is enabled, (3) mesh elements are refined as needed to obtain sufficient resolution for new nuclei. (4) The nodal solution vector for the order parameter is explicitly modified to reflect the formation of the new nuclei. (5) The time step size is modified as needed. Upon the completion of the nucleation step, the next phase field time step is executed. 
The nucleus seeding method described in this paper differs significantly from that described in Refs. [29, 30]. Unlike that method, the concentration field is left unchanged, and thus mass conservation is naturally met. OPO nucleus seeding is more computationally efficient and significantly easier to implement because it eliminates the need to calculate mass conservation requirements before nucleus introduction. Figure 2 illustrates how the concentration and structural order parameter fields are altered in the OPO seeding method for initially uniform fields. Upon determination of a new nucleus position, an OPO nucleus is introduced by altering the value of the structural order parameter field to reflect the presence of the second phase (Fig. 2a). The OPO nucleus introduced into the simulation domain must be larger than the critical nucleus size for the given solute concentration. Considerations for the choice of the size of the introduced nuclei are given in the Results and Discussion. Each nucleus has a finite size and encompasses multiple elements such that the interface is well-resolved. Although we choose a circular nucleus with a diffuse interface profile, alternative nucleus shapes such as those described in Refs. [36--38] could be incorporated. The modification of the order parameter field may either be carried out for only one time step (when a new nucleus is seeded), or be held fixed for several time steps; both cases are studied in this paper.

For comparison purposes, we use the nucleus seeding method in Ref. [29] as the initial condition for a single-particle simulation by altering both the concentration and the structural order parameter fields (hereafter referred to as "order parameter and concentration" or OPC seeding). The treatment of the structural order parameters is the same for both OPO seeding and OPC seeding (Fig. 2a). However, the solute concentration is increased in the nucleus and depleted in the surrounding region for OPC seeding (Fig. 2c). The total quantity of solute in the computational domain is the same for OPO and OPC seeding. In this work, the depletion region width and concentration is determined in a fashion similar to Ref. [29], but we choose to minimize the width of the depletion region, $w_{d}$, by setting the concentration in the depletion region, $c_{d}$, close to zero due to the low initial 


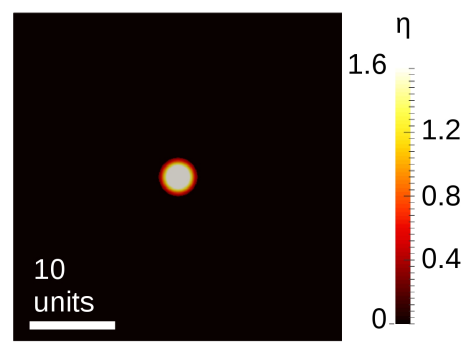

(a)

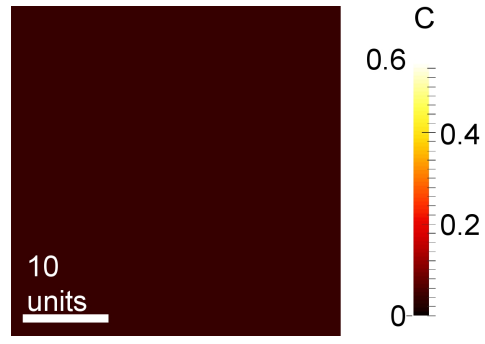

(b)

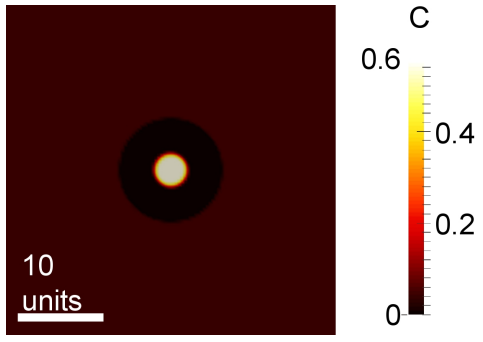

(c)

Figure 2: The concentration and structural order parameter profiles upon nucleus seeding. (a) The structural order parameter field for both an OPO seed and an OPC seed. (b) The concentration field for an OPO seed, which is uniform. (c) The concentration field for an OPC seed, which is non-uniform.

solute concentration. The amount of solute that must be compensated for by the reduced concentration in the depletion region, $\Delta s$, is calculated from the intial concentration, the system volume, and the nucleus volume and composition. We then estimate $w_{d}$ as the width that is needed to compensate for $\Delta s$ assuming $c_{d}=0$. For simplicity, we round $w_{d}$ up to the nearest integer, and adjust $c_{d}$ such that the amount of solute is identical in both the OPO and OPC simulations.

\subsection{Simulation conditions}

We present the results from two sets of simulations designed to explore the OPO nucleation algorithm. The first set is performed to characterize the evolution of a single OPO nucleus. The second set is performed to analyze the phase transformation kinetics predicted in the form of the Avrami equation [47--49]. We perform an Avrami analysis similar to that described in Ref. [29] to study concurrent nucleation and growth with a constant nucleation rate. In addition, we determine whether mesh and/or time adaptivity has an effect on the results.

In this work, simulations are performed in two dimensions. No-flux boundary conditions are applied for the concentration and structural order parameters, and the initial hydrogen concentration is set to $c=0.0562$, which is less than the concentration used in Ref. [40] but high enough to induce concurrent nucleation and growth. The equilibrium 
interface width is 1.33 in nondimensionalized units measured between $0.1 \eta_{p}<\eta<0.9 \eta_{p}$, where $\eta_{p}$ is the value of $\eta$ in the hydride. Introduced nuclei have a radius of $r=1.8$, which is twice the minimum stable particle size at this composition so that nuclei will grow even if introduced into regions with decreased supersaturation. The diffuse interface width of the nuclei is 0.9 and the profile is specified by a sine function, although a different profile and width could be chosen. The so-called BDF2 implicit time integration scheme [50], which refers to the second-order accurate backward differentiation formula (BDF), is employed. The nonlinear relative convergence tolerance is $10^{-8}$. The simulations use a single matrix preconditioner and the Additive Schwarz Method preconditioning process. For simulations with a fixed time step size, a nondimensionalized time step size of $\Delta t=0.01$ is used. For simulations with time adaptivity, computational efficiency is optimized by employing a variable time step size based on the ratio of the time step size to the CPU time required to iteratively solve the system of equations for each time step [45]. A maximum of $1 \%$ increase per time step is allowed, and the maximum $\Delta t=1.0$ is chosen. Upon a nucleation event, the time step is reduced to $\Delta t=0.01$. The mesh is composed of square, four-node quadrilateral elements and linear Lagrange shape functions are used for the structural order parameter, concentration, and chemical potential variables. Simulations without mesh adaptivity are performed with an element side length of 0.32 . Simulations with adaptive meshing have a maximum element side length of 5.12 and a minimum element side length of 0.32 , corresponding to a maximum of four levels of refinement. Adaptive mesh refinement involves splitting and refining elements based on an error indicator. We use the gradient jump indicators [51,52] for $c$ and $\eta$, which computes the change in the gradient from one element to the next. The mesh is refined for any element within $75 \%$ of the maximum jump, and the mesh is coarsened for any element within $5 \%$ of the minimum jump, where the maximum and minimum are taken over the entire mesh. These refinement parameters are chosen to ensure that introduced nuclei are appropriately resolved. 
Single-particle simulations are performed with a nondimensionalized domain size of $38.4 \times 38.4$ to study the evolution of OPO and OPC nuclei. The structural order parameter field initially contains one seed in the center of the computational domain. The OPC nucleus has a concentration of $c=0.59$, the depletion region has a concentration of $c_{d}=3.13 \times 10^{-4}$ and a depletion width of $w_{d}=8$, and the matrix outside the depletion region has a concentration of $c=0.0562$. No mesh or time adaptivity are used for the single-particle simulations.

The equilibrium phase fraction for the given concentration is needed for the Avrami analysis. The equilibrium phase fraction is found by allowing a planar interface simulation with a domain size of $76.8 \times 9.6$ to achieve equilibrium, where equilibrium is defined as uniform chemical potential within convergence tolerance. The initial condition for the simulation is an OPO hydride plate at the left edge of the computational domain and a uniform concentration of $c=0.0562$. No mesh adaptivity is used, but time adaptivity is employed to attain equilibrium efficiently.

The Avrami analysis simulations are performed with a nondimensionalized domain size of $460.8 \times 460.8$. The values $\tilde{k}_{1}=2.5 \times 10^{-4}$ and $k_{2}=4.71 \times 10^{-2}$ are chosen to induce concurrent nucleation and growth conditions with a constant nucleation rate. The value of $k_{2}$ controls the nucleation rate by affecting the driving force for nucleation, while the value of $\tilde{k}_{1}$ controls the number of nuclei formed during the early stage of the simulation [29]. Finally, the structural order parameter field initially contains one randomly located nucleus. This initial condition is chosen based on numerical considerations; by introducing a nucleus, the residual of the initial condition is large enough such that the relativetolerance convergence criterion is met. 


\section{Results and discussion}

\subsection{OPO nucleus evolution}

To assess the OPO nucleus seeding method, the evolution of an OPO nucleus is compared to that of an OPC nucleus. The values of the concentration and structural order parameter fields at the center of the nucleus, denoted as $c_{p 0}$ and $\eta_{p 0}$, respectively, are used to quantify the evolution of an OPO nucleus. Figure 3 shows $c_{p 0}$ and its derivative with respect to time, which indicate how solute accumulates in the precipitate during nucleus formation. Initially $c_{p 0}$ increases rapidly; this behavior is hereafter termed the "solute accumulation" (SA) stage. As $c_{p 0}$ approaches the equilibrium concentration, solute accumulation slows, with $\partial c_{p 0} / \partial t$ decreasing below 1.0 at $t=0.16$, which we hereafter refer to as $t_{c}$. During the SA stage, a solute depletion region with a smooth gradient profile is naturally formed around the nucleus. Note that the equilibrium value of $c_{p 0}$ has not yet been attained during the time examined, but the plot range is selected to focus on the early transient behavior that occurs immediately following nucleus introduction. In addition, $\eta_{p 0}$ and its time derivative are plotted in Fig. 4. The value of $\eta_{p 0}$ decreases during the SA stage and exhibits a minimum at $t=0.08$, hereafter referred to as $t_{\text {min }}$, but subsequently increases rapidly, with $\partial \eta_{p 0} / \partial t$ attaining a maximum at $t=0.15$. The value of $\eta$ in the precipitate is slightly higher than its equilibrium value during the time examined, but the value decreases as the fraction of the precipitate phase evolves toward its equilibrium value. This behavior of $\eta$ in the precipitate is a result of the shape of the free energy well shown in Fig. 5, and will in general depend on the details of the specific free energy function.

The behavior of $c_{p 0}$ and $\eta_{p 0}$ can be elucidated by examining the free energy of the introduced seed. Figure 5 is a contour plot of $f_{c h e m}$ with the $\left(c_{p 0}, \eta_{p 0}\right)$ path indicated by the red squares. The OPO nucleus has a much higher free energy density than either the matrix or precipitate phases as it is initialized (the leftmost square). As such, there is a 
large driving force to diffuse solute into the nucleus. Once the presence of additional solute has lowered the free energy of the nucleus, further energy reduction occurs by the increase of $\eta_{p 0}$ toward its equilibrium value.

While OPO seeding naturally generates a nucleus with a surrounding depletion region, the initial SA stage will delay the precipitate growth as compared to an OPC nucleus, which seeds both concentration and order parameter fields. We first examine the $\left(c_{p 0}, \eta_{p 0}\right)$ path of an OPC nucleus, shown by the orange triangles in Fig. 5. Although the OPC nucleus is near the energetic minimum, its $c_{p 0}$ and $\eta_{p 0}$ values evolve slightly following the introduction of the seed. Figure 6a shows the precipitate area fractions of an OPO and an OPC nucleus. The area is found by thresholding the structural order parameter field at $\eta=1.5$ and summing the area of each element within the thresholded region. The area of the OPO nucleus is smaller than the area of the OPC nucleus at any given time over the time simulated. The maximum difference in area is $9 \%$ at $t=1.8$ and the difference decreases slowly after that to $4 \%$ at $t=10$. Growth of the OPO nucleus may be delayed both due to the time needed to accumulate solute and due to the initial decrease in the structural order parameter, which lowers the driving force for solute accumulation.

To maintain the driving force and to prevent nucleus dissolution, the structural order parameter may be held fixed during the SA stage (termed an "OPO+hold" seed). The hold time, $t_{\text {hold }}$, can be found by examining the evolution of $c_{p 0}, \eta_{p 0}$, and the area fraction of the OPO seed. The area fractions of an OPO+hold nucleus and an OPC nucleus are compared for a given $t_{\text {hold }}$, and the $t_{\text {hold }}$ value that yields the smallest maximum difference in area fractions is chosen. The characteristic times $t_{c}$ and $t_{\min }$ provide a guideline for choosing $t_{\text {hold }}$. Figure 6a shows the area fraction of an OPO+hold nucleus with $t_{\text {hold }}=t_{\text {min }}$. The maximum difference in the area fraction for the OPO+hold nucleus versus the OPC nucleus is approximately $4 \%$ at $t=2.8$ and the difference decreases slowly after that to $1.6 \%$ at $t=10$. The difference is significantly less than the difference between the OPO nucleus and the OPC nucleus. For the cases examined, the area fraction of the OPO+hold 


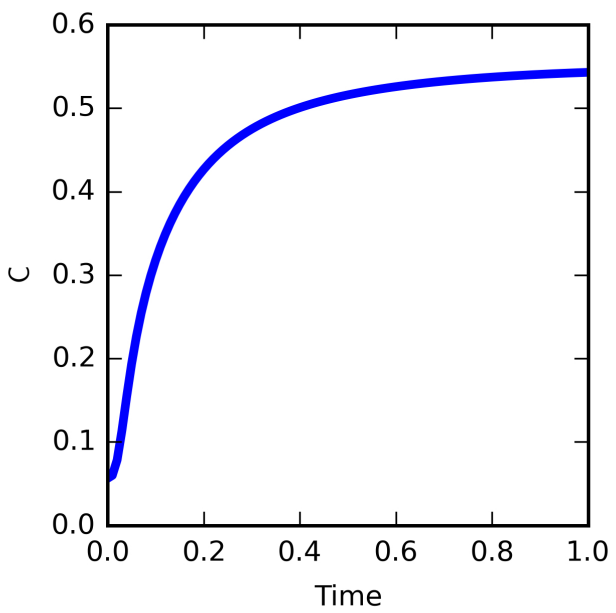

(a)

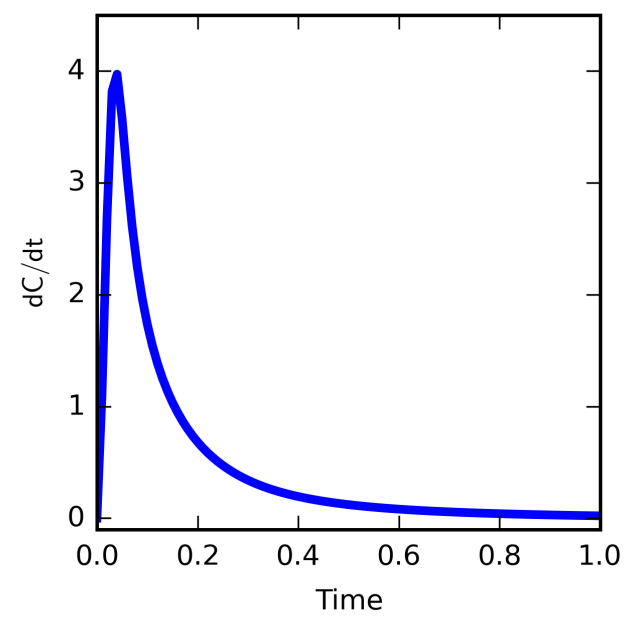

(b)

Figure 3: (a) The value of the concentration field at the center of the nucleus for OPO seeding without order parameter holding. (b) The rate of change of the concentration value at the center point with respect to time. 


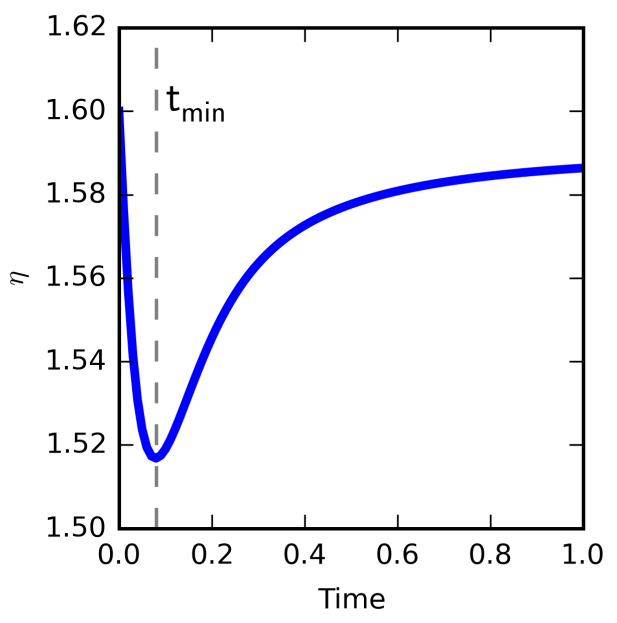

(a)

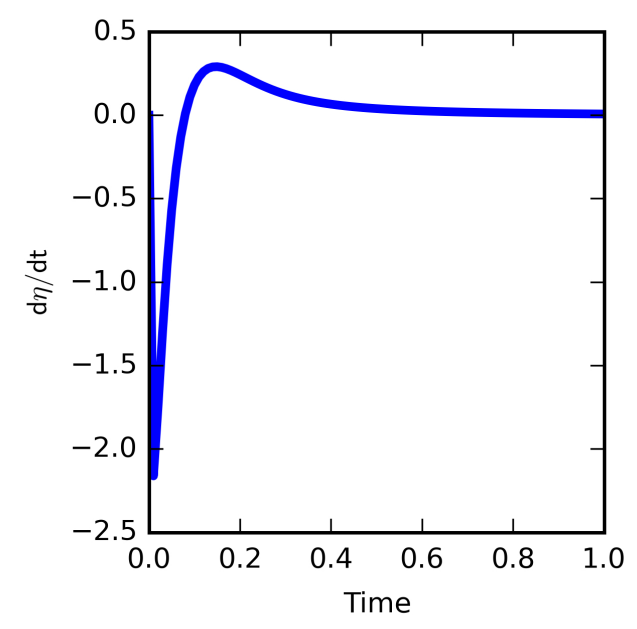

(b)

Figure 4: (a) The value of the structural order parameter field at the center of the nucleus for OPO seeding without order parameter holding. (b) The rate of change of the structural order parameter value at the center point with respect to time. 


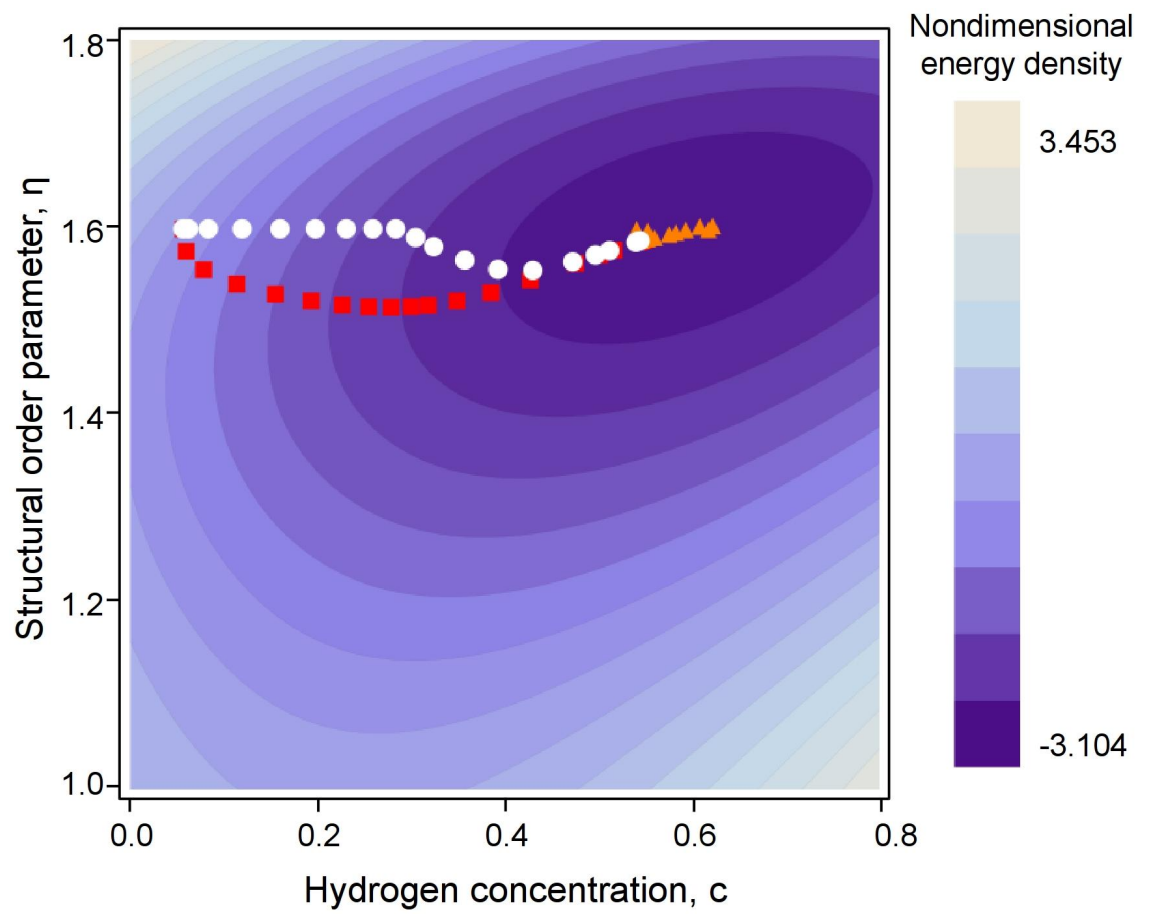

Figure 5: The contour plot of $f_{\text {chem }}$ and the $\left(c_{p 0}, \eta_{p 0}\right)$ paths for the different nucleus seeds. The red squares are for the OPO nucleus and the white circles are for the OPO+hold nucleus; in both cases the energy of the as-seeded nucleus is indicated by the leftmost point. The orange triangles are for the OPC nucleus.

nucleus is insensitive to $t_{\text {hold }}$ values between $t_{\min }$ and $t_{c}$, and we believe this finding is likely general. This insensitivity is beneficial when using time adaptivity in simulations. A comparison of $\eta_{p 0}$ for the OPO nucleus and the OPO+hold nucleus is shown in Fig. 6b; the undershoot of $\eta_{p 0}$ is much less in the OPO+hold case. In addition, the $\left(c_{p 0}, \eta_{p 0}\right)$ path for the OPO+hold nucleus is shown in Fig. 5 by the white circles. During the time that the structural order parameter is held fixed, the $\left(c_{p 0}, \eta_{p 0}\right)$ path remains where the driving force for solute accumulation is larger than that followed by the OPO nucleus, accelerating the stabilization of the OPO+hold nucleus.

The value of $t_{\min }$ varies depending on the simulation setup, and thus the value chosen for $t_{\text {hold }}$ should be adjusted accordingly. However, the hold time should not be longer than necessary because it may delay growth by preventing the interface from propagating even under growth conditions. The value of $t_{\text {min }}$ may vary depending on the solute mobility and the kinetic coefficient of the structural order parameter, the driving force of 
the transformation, the local solute concentration, and the size of the seeded nucleus. For example, a lower solute concentration will likely require a longer hold time to accumulate solute.

The size of the introduced nucleus will vary depending upon the model system and the simulation parameters, and thus needs to be adjusted accordingly. The nucleus size can be determined based on the results of single-particle simulations for each system. The introduced nucleus should be as small as possible without dissolving. The critical nucleus size, which is typically on the order of a nanometer, presents a lower bound on the nucleus size and may be determined by classical nucleation theory. However, the size of the introduced nucleus is also affected by the maximum mesh resolution of the simulation and the diffuse interface width, which are numerical parameters. Unless the critical nucleus size is resolved by the simulation mesh, the introduced nucleus size should be larger than the diffuse interface width to ensure that a bulk region exists. If the introduced nuclei are much larger than the critical size and the time to grow to that size is significant, a time correction will be necessary. In this work, the critical radius is sufficiently small that this correction is unnecessary. We also note that a larger critical radius will require a larger $t_{\text {hold }}$ for a given system, and thus these parameters need to be consistently determined.

\subsection{Validation of OPO nucleation and growth}

The early-stage kinetics of precipitation and growth simulated with the OPO nucleation algorithm are analyzed by fitting the simulation results to the Avrami equation [47--49]. For these simulations, we do not use time or mesh adaptivity; these simulations have the highest numerical resolution and accuracy considered in this work. Nine simulations, denoted with the subscript " 0, " are performed with different seeds for random number generation to obtain statistical information. We use a hold time of $t_{\text {hold }}=1.25 t_{\text {min }}=0.1$.

First, several overall observations regarding the number of precipitates and the area 


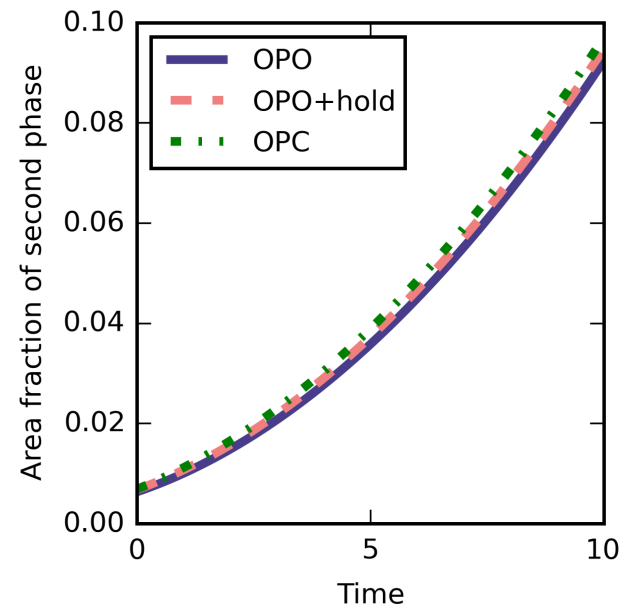

(a)

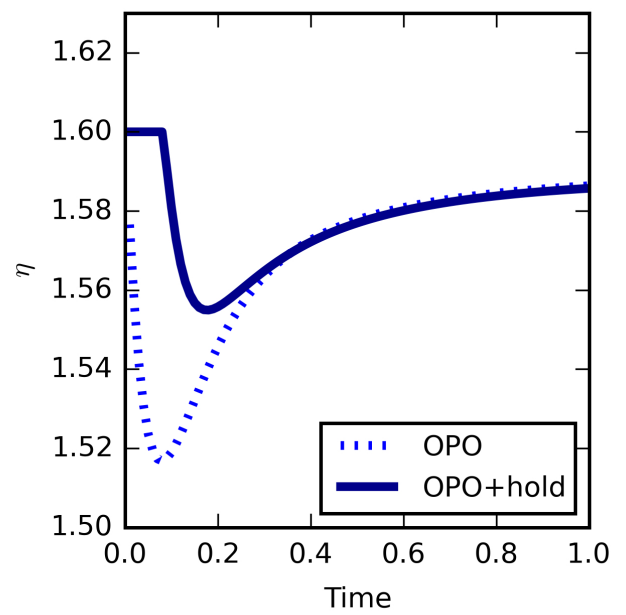

(b)

Figure 6: (a) The area fraction of the second-phase particle using OPO, OPO+hold, and OPC methods. (b) The value of the structural order parameter at the center of an OPO nucleus and at the center of an OPO+hold nucleus with $t_{\text {hold }}=t_{\min }=0.08$.

fraction of the transformed phase are made. Figure 7a presents the snapshots of one of the nine simulations performed, showing the distribution of precipitates with varying size as a result of concurrent nucleation and growth. For the set of simulations, approximately 30 nuclei form by $t=2$. By $t=20$, nucleation is almost complete, and approximately 200 nuclei have formed. 

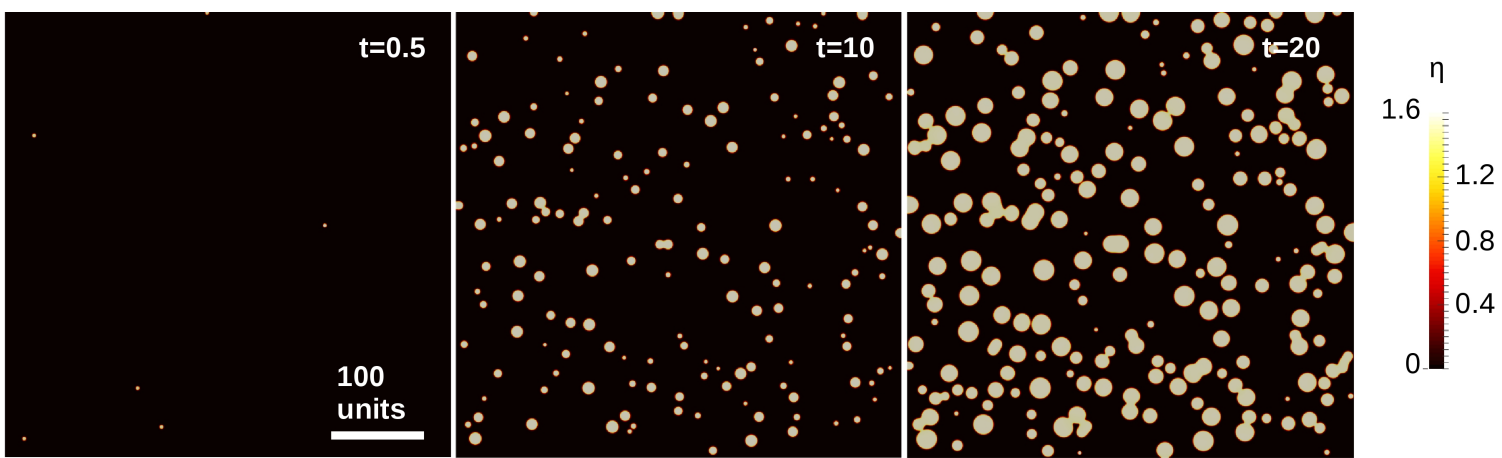

(a)
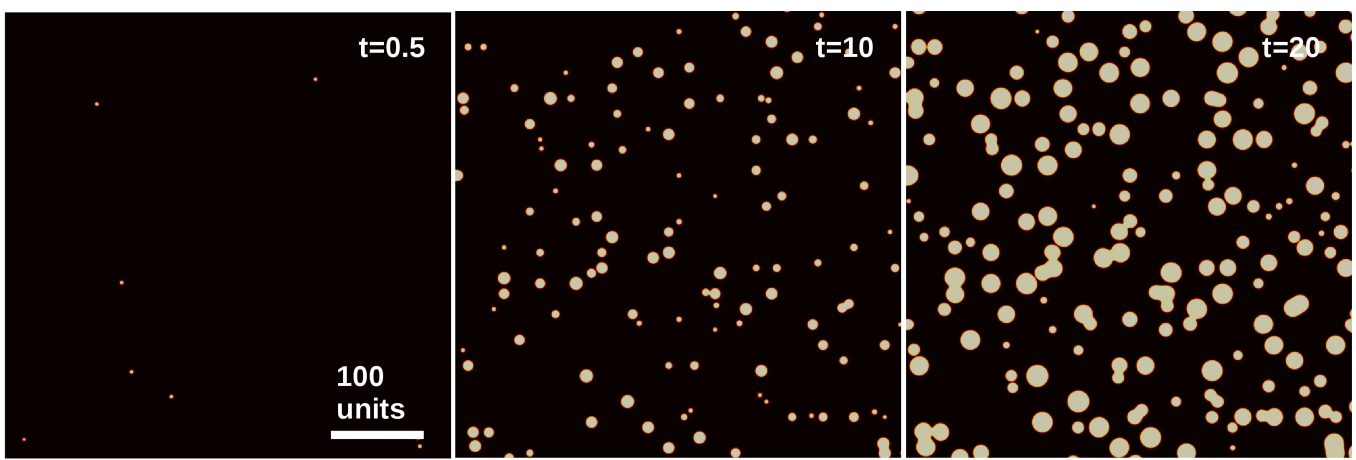
1.6

(b)

Figure 7: Structural order parameter field shown concurrent nucleation and growth (CNG) conditions (a) with no mesh or time adaptivity used for the simulation and (b) with mesh and time adaptivity used.

The area fraction of the transformed phase, $A(t)$, for the fastest and slowest transformations are shown in Fig. $8 \mathrm{a}$. The area fraction varies by $20 \%$ at $t=20$ between the slowest and fastest transformations. This variation is due to the stochastic nature of the nucleation algorithm, which causes the exact location and timing of the nucleation events to be different for each simulation.

To confirm that the $\mathrm{OPO}+$ hold algorithm and implementation reproduce the results expected from classical nucleation theory, we consider the Avrami equation

$$
A(t) / A_{f}=1-\exp \left(-K t^{m}\right)
$$

where $t$ is time, $A_{f}$ is the equilibrium area fraction, $m$ is the Avrami coefficient, which indicates the type of transformation kinetics, and $K$ is the time constant. To perform the 


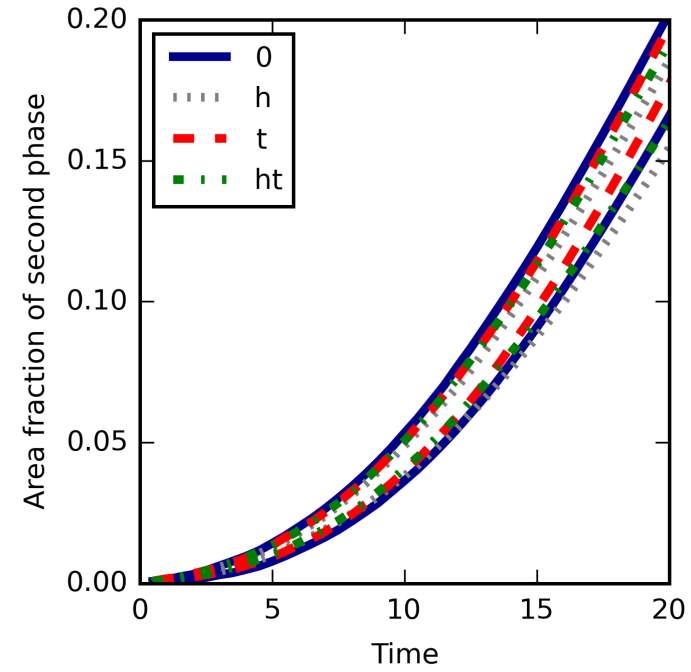

(a)

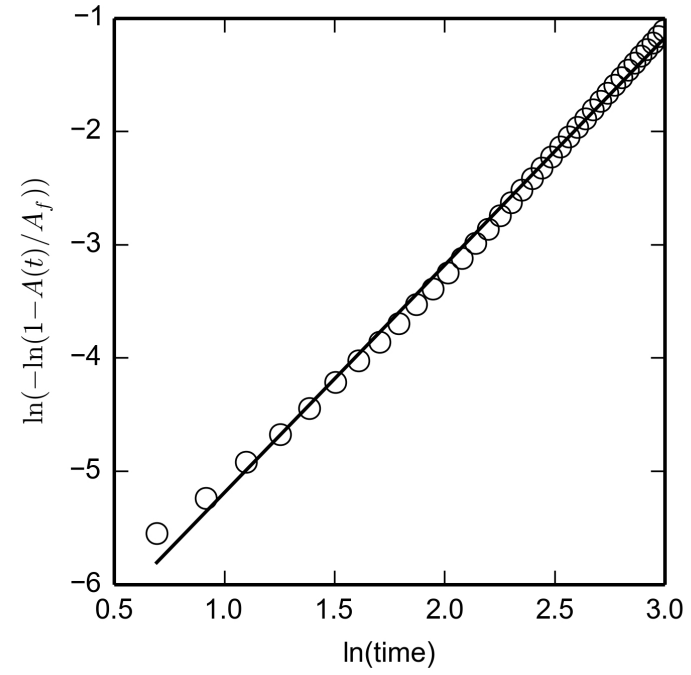

(b)

Figure 8: (a) The area fraction of the transformed phase is plotted for the fastest and slowest cases within each set of simulations performed with no adaptivity (solid, 0 ), with mesh adaptivity (gray dots, h), with time adaptivity (red dash, $t$ ), and both mesh and time adaptivity (green dash-dot, ht). (b) An example of an Avrami fit for the area fraction of the transformed phase (the data is indicated by the circles and the fit is the solid line).

Avrami analysis, Eq. 12 is rewritten as

$$
\ln \left[-\ln \left(1-A(t) / A_{f}\right)\right]=\ln K+m \ln (t) .
$$

The value of $A(t) / A_{f}$ is inserted into the left-hand side of Eq. 13, and the resulting values are fit to the right-hand side of Eq. 13, such that $m$ and $K$ are determined from the slope and the intercept of the line, respectively. An example is given in Fig. 8b. Although there is some deviation of the data from the single linear fit, the fit provides simple a quantitative metric of the phase transformation kinetics and the effects of mesh and/or time adaptivity on it. Because the slope varies over the time period examined, the value obtained from the linear fit is an average of the slope over that time period. The time constant $K$ is found by calculating the value of the linear fit when $\ln (t)=0$. In this work, $m$ and $K$ are calculated using $A(t)$ between $2 \leq t \leq 20$, while nucleation and growth are ongoing. As we mentioned previously, for a given initial condition, the stochastic 
nature of the nucleation algorithm will generate a range of transformation kinetics. This variation is quantified by the Avrami coefficient and time constant averaged over the nine simulations, $\bar{m}$ and $\bar{K}$, respectively, and their standard deviations, $\sigma_{m}$ and $\sigma_{K}$. For the validation simulations, we find the values $\bar{m}_{0}=2.01, \sigma_{m, 0}=0.0370, \bar{K}_{0}=7.44 \times 10^{-4}$, and $\sigma_{K, 0}=1.18 \times 10^{-4}$.

The OPO nucleation algorithm allows for a broad range of nucleation conditions to be modeled. By varying $\tilde{k}_{1}$ and $k_{2}$ in Eq. 10, more nuclei may be formed earlier in the simulation, resulting in $m<2$ in two dimensions. Site saturation conditions, in which all nuclei are present at the start of the phase transformation, result in $m=1$ in two dimensions. Simmons et al. reported difficulty in lowering the Avrami coefficient much below 1.67 due to numerical instabilities from the composition profiles of the nucleus and depletion region [29]. The OPO nucleation seeding method gives no such instability because it does not introduce sharp concentration gradients. By increasing $\tilde{k}_{1}$ by one order of magnitude, $m$ decreases to 1.25 . Further increasing $\tilde{k}_{1}$ should result in site saturation, indicating the flexibility of this algorithm at modeling different nucleation behavior.

\subsection{Effect of adaptivity on concurrent nucleation and growth simulations}

Large-scale three-dimensional phase field simulations of concurrent nucleation and growth are computationally intensive. To improve computational efficiency, time and mesh adaptivity may be used to reduce resolution while retaining sufficient accuracy. The time adaptivity scheme ensures a small time step during nucleation events but allows it to grow in the absence of nucleation. Mesh adaptivity reduces the degrees of freedom by using larger mesh elements in regions with lower field gradients; an example of the mesh adaptivity within the simulation is shown in Fig. 9. However, the effect of the reduced resolution must be evaluated, and sufficient mesh and time resolution must be retained within the simulation. For example, if a mesh is overly coarse with respect to the characteristic length scale of the microstructure, the spatial distribution of precipitates may 

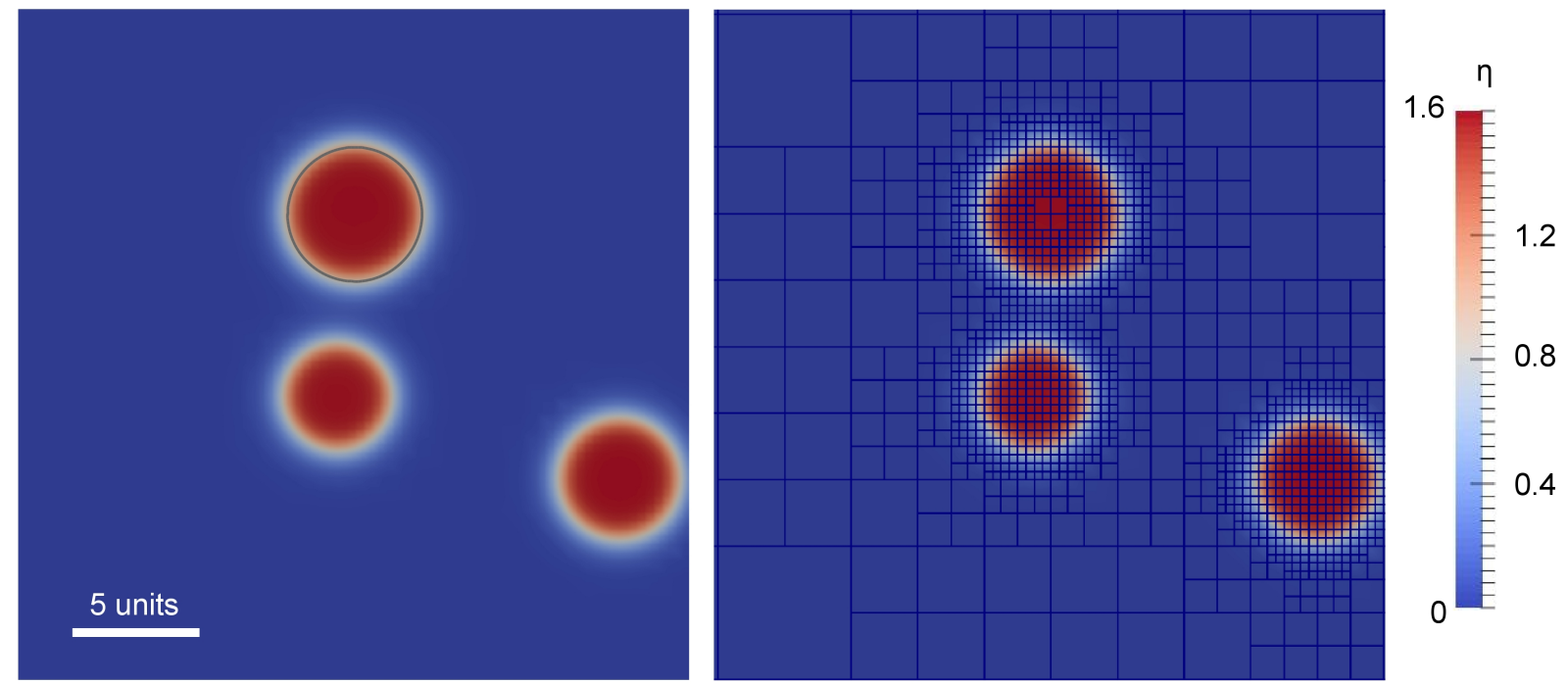

Figure 9: A magnified snapshot of a concurrent nucleation and growth simulation performed with mesh adaptivity. Left: Three precipitates are shown. A perfect circle has been imposed over the topmost precipitate (dashed gray line) as a guide for the eye. Right: The same snapshot, but with the mesh shown in white, which contains both the largest and smallest mesh elements. The mesh is finest at the precipitate interfaces.

be affected. If the time step is too large for the nucleation rate, the assumption that only one nucleus may form per element may be violated, affecting the number of nuclei introduced into the simulation. While mathematical analyses to determine the upper limit of temporal and spatial discretization steps for nucleation and growth are beyond the scope of this paper, we present a method to quantify the effect of adaptivity on the simulation results. We perform simulations with mesh adaptivity, time adaptivity, and combined mesh and time adaptivity, denoted with the subscripts " $h$, ," "t $t$," and "ht," respectively. As before, nine simulations are performed for each case to capture the statistical behavior.

Following the discussion in Section 3.2, we examine the microstructure and the transformation kinetics simulated when using adaptivity. Figure $7 \mathrm{~b}$ shows the snapshots of a concurrent nucleation and growth simulation performed with both mesh and time adaptivity; the initial condition is identical to that in Fig. 7a. The microstructure is similar to the previous case presented in Fig. 7a, although for this specific simulation, there are fewer precipitates at $t=20$. To examine the kinetics, the area fraction of the transformed phase is shown in Figure 8 for the fastest and slowest cases for each set of simulations. 
The area fractions for simulations with different adaptivities are similar to those of the simulations with no adaptivity. Therefore, for the given adaptivity parameters, time or mesh adaptivity do not appear to affect the simulated kinetics.

We perform the Avrami analysis described in Section 3.2 to quantify the transformation kinetics obtained from simulations that utilize adaptivity, and means and standard deviations are shown in Fig. 10. As presented in Table 1, the values of $\bar{m}_{t}, \bar{m}_{h}$, and $\bar{m}_{h t}$ are equal to $\bar{m}_{0}$ within two significant figures, and $\sigma_{m} / \bar{m}$ values are in the range of 0.018 to 0.036 , indicating a narrow distribution. Mesh adaptivity results in the largest $\sigma_{m}$ $\left(\sigma_{m, h} \approx 1.9 \sigma_{m, 0}\right)$, while time adaptivity and combined time and mesh adaptivity result in similar $\sigma_{m}\left(\sigma_{m, t} \approx \sigma_{m, h t} \approx 1.3 \sigma_{m, 0}\right)$. These results indicate that the power-law exponent in Eq. 12 is insensitive to both the stochastic nature of nucleation and to adaptivity. In contrast, the time constant $K$ exhibits more variation from simulation to simulation within each set. The values of $\bar{K}_{h}$ and $\bar{K}_{t}$ differ from $\bar{K}_{0}$ by more than $10 \%$, with the largest differences in $K$ occurring when using mesh adaptivity. These values also exhibit larger scatter, as evidenced by $\sigma_{K} / \bar{K}$ in the range of 0.16 to 0.24 . This greater variation indicates that $K$ is sensitive to the details of when and where nucleation events occur. Overall, the case with no adaptivity results in the smallest values of $\sigma_{m}$ and $\sigma_{K}$, showing that adaptivity tends to increase the variation in the simulated kinetics. However, the overlap in the error bars between the data sets indicate that no systematic differences are evident for differences in the average $m$ and $K$ values.

Table 1: Average kinetic coefficients and their standard deviations for the concurrent nucleation and growth simulations. Listed are the average Avrami coefficient and its standard deviation, $\bar{m}$ and $\sigma_{m}$, respectively, and the average time constant and its standard deviation, $\bar{K}$ and $\sigma_{K}$, respectively.

\begin{tabular}{|c|c|c|c|c|}
\hline Adaptivity type & $\bar{m}$ & $\sigma_{m}$ & $\bar{K}$ & $\sigma_{K}$ \\
\hline \hline no adaptivity & 2.01 & 0.0370 & $7.44 \times 10^{-4}$ & $1.18 \times 10^{-4}$ \\
\hline mesh adaptivity & 2.02 & 0.0719 & $6.53 \times 10^{-4}$ & $1.59 \times 10^{-4}$ \\
\hline time adaptivity & 1.98 & 0.0499 & $8.15 \times 10^{-4}$ & $1.45 \times 10^{-4}$ \\
\hline mesh and time adaptivity & 1.97 & 0.0479 & $7.79 \times 10^{-4}$ & $1.28 \times 10^{-4}$ \\
\hline
\end{tabular}




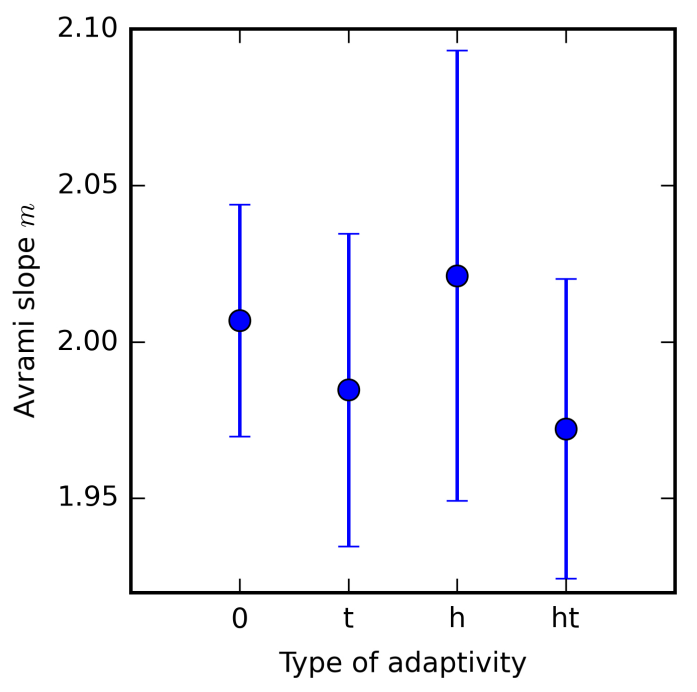

(a)

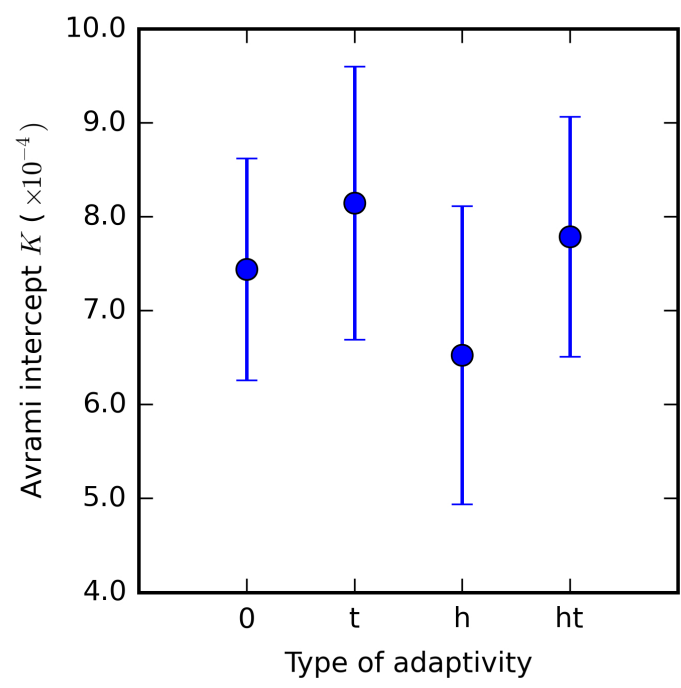

(b)

Figure 10: The average kinetic coefficients and one standard deviation (shown by the error bars) for the concurrent nucleation and growth simulations performed with no adaptivity (0), with mesh adaptivity (h), with time adaptivity ( $\mathrm{t}$ ), and both mesh and time adaptivity (ht). The information regarding the Avrami slope $m$ is shown in (a) and the Avrami intercept $K$ is shown in (b). 


\section{Conclusion}

The explicit nucleation method described in this paper is a refinement of the method developed by Simmons et al. [29, 30]. In our proposed method, OPO nucleation, a nonconserved structural order parameter is locally altered to introduce a nucleus in a coupled conserved-nonconserved phase field model. The OPO nucleus seeding method induces the formation of both a nucleus and a depletion region for the conserved variable, while naturally satisfying conservation rules. Mass conservation is naturally observed via the evolution equation instead of explicitly accounted for during nucleus introduction, simplifying the simulation code and allowing a depletion region to be formed with a profile controlled by the model physics. In addition, numerical instabilities are avoided by eliminating sharp field gradients. The implementation details of the OPO nucleation algorithm in a finite element framework are presented, although the algorithm could be used with other numerical methods such as the finite difference method.

Upon introduction of an OPO nucleus, three processes occur concurrently: (a) a solute accumulation stage, in which solute diffuses to the nucleus, (b) the formation of a solute depletion region around the nucleus, and (c) a decrease in the structural order parameter. We describe two characteristic times within the solute accumulation stage that are based on the evolution of the conserved and nonconserved variables. The characteristic time for the conserved order parameter is the time at which solute accumulation slows significantly, and the characteristic time for the conserved order parameter identifies the time at which the structural order parameter value is minimal. In addition, the solute accumulation stage causes a precipitate formed by OPO seeding to be slightly smaller at a given time than one seeded with the appropriate values of both concentration and structural order parameter fields. However, this discrepancy can be reduced by holding the structural order parameter value fixed during this stage. For the cases examined, we find that the area fraction of the $\mathrm{OPO}+$ hold nucleus is insensitive to the hold time for values between 
the the two characteristic times mentioned above. The evolution of an OPO nucleus is explained based on the free energy landscape.

The early-stage kinetics of precipitation and growth simulated with the OPO nucleation algorithm are analyzed by fitting the simulation results to the Avrami equation. With an increased nucleation rate coefficient, we obtained an Avrami coefficient as low as 1.25. A statistical analysis is performed to study the effect of mesh and/or time adaptivity on the simulated kinetics. We find the results are comparable between these data sets with different mesh and/or time adaptivity.

The explicit nucleation method presented here is general and can be straightforwardly applied to other materials systems that are described by a coupled conservednonconserved phase field model. However, the evolution behavior of an OPO nucleus must be analyzed to identify the hold time required, if necessary. In addition, the results should be analyzed to ensure sufficient spatial and temporal resolution with or without adaptivities. This paper provides the framework for determining the adaptivity parameters, as well as the hold time.

\section{Acknowledgements}

This research was supported by the Consortium for Advanced Simulation of Light Water Reactors (www.casl.gov), an Energy Innovation Hub (http://www.energy.gov/ hubs) for Modeling and Simulation of Nuclear Reactors under U.S. Department of Energy Contract No. DE-AC05-00OR22725. The simulations were performed using the high

performance computation resources Fission and Quark at Idaho National Laboratory (INL). Many thanks to the MOOSE team at INL for the continuing, generous help and support with MOOSE. Finally, many thanks to Michael Tonks at INL for his help and experience with phase field modeling using MOOSE.

\section{Appendix}

The parameters for the phase field model are given in nondimensionalized units. The nondimensionalization of the model is given as follows. A nondimensionalized quantity $q$ in the phase field model has a corresponding dimensional quantity $\hat{q}$. Characteristic scale values for the system's length, energy, and time are defined, and the equations 
are nondimensionalized using these values. The characteristic length scale is set to $\hat{l}=$ $1 \times 10^{-9} \mathrm{~m}$ because we focus on nanoscale phenomena in our applications. Reference [40] indicates that the free energy functional was fit to the Zr-H phase diagram at 523 $\mathrm{K}$. Thus, the characteristic volumetric energy scale is chosen as $|\Delta \hat{f}|=1 \times 10^{8} \mathrm{~J} / \mathrm{m}^{3}$, on the order of volumetric energy of mixing for the $\mathrm{Zr}-\mathrm{H}$ system [53]. The atomic volume of zirconium is $\Omega_{0}=1.4 \times 10^{-5} \mathrm{~m}^{3} / \mathrm{mol}$. While no experimental information exists for the Allen-Cahn kinetic coefficient, $\hat{L}=1 \mathrm{~m}^{3} \mathrm{~J}^{-1} \mathrm{~s}^{-1}$ is chosen to maintain a diffusioncontrolled transformation [54]. Therefore, the characteristic time is $\hat{t}_{0}=2.86 \times 10^{-4} \mathrm{~s}$. The relationships between the dimensional and dimensionless quantities are presented in Table 2.

Table 2: Nondimensionalization expressions for quantities within the phase field model.

\begin{tabular}{|c|c|}
\hline Quantity & Nondimensionalization expression \\
\hline \hline Time & $t=\hat{t} \hat{L}|\Delta \hat{f}|$ \\
\hline Length & $r=\hat{r} / \hat{l}$ \\
\hline Hydrogen mobility & $M=\hat{M} \Omega_{0} /\left(\hat{l}^{2}|\Delta \hat{f}|\right)$ \\
\hline Allen-Cahn kinetic coefficient & $L=\hat{L}|\Delta \hat{f}| \hat{t}_{0}$ \\
\hline Gradient coefficient & $\kappa=\hat{\kappa} /\left(|\Delta \hat{f}| \cdot \hat{l}^{2}\right)$ \\
\hline Chemical energy & $f_{\text {chem }}=\hat{f}_{\text {chem }} /|\Delta \hat{f}|$ \\
\hline
\end{tabular}

[1] D. Porter and K. Easterling, Phase Transformations in Metals and Alloys. Chapman \& Hall, 1992.

[2] A. Jain, S. P. Ong, G. Hautier, W. Chen, W. D. Richards, S. Dacek, S. Cholia, D. Gunter, D. Skinner, G. Ceder, et al., "Commentary: The materials project: A materials genome approach to accelerating materials innovation," APL Materials, vol. 1, no. 1, p. 011002, 2013.

[3] P. Patel, "Materials genome initiative and energy," MRS Bulletin, vol. 36, pp. 964--966, 122011.

[4] Y. Kwon, K. Thornton, and P. W. Voorhees, "Coarsening of bicontinuous structures via nonconserved and conserved dynamics," Physical Review E, vol. 75, no. 2, p. 021120, 2007.

[5] Y. Kwon, K. Thornton, and P. Voorhees, "The topology and morphology of bicontinuous interfaces during coarsening," EPL (Europhysics Letters), vol. 86, no. 4, p. 46005, 2009.

[6] D. Seol, S. Hu, Y. Li, J. Shen, K. Oh, and L. Chen, "Three-dimensional phase-field modeling of spinodal decomposition in constrained films," Metals and Materials International, vol. 9, no. 1, pp. 61--66, 2003. 
[7] J. W. Cahn, “On spinodal decomposition," Acta Metallurgica, vol. 9, no. 9, pp. 795--801, 1961.

[8] L.-Q. Chen and A. Khachaturyan, "Computer simulation of structural transformations during precipitation of an ordered intermetallic phase," Acta Metallurgica et Materialia, vol. 39, no. 11, pp. 2533--2551, 1991.

[9] S. Hu and L. Chen, "A phase-field model for evolving microstructures with strong elastic inhomogeneity," Acta Materialia, vol. 49, no. 11, pp. 1879--1890, 2001.

[10] J. Zhu, T. Wang, A. Ardell, S. Zhou, Z. Liu, and L. Chen, "Three-dimensional phasefield simulations of coarsening kinetics of $\gamma^{\prime}$ particles in binary ni-al alloys," Acta Materialia, vol. 52, no. 9, pp. 2837--2845, 2004.

[11] H.-J. Diepers, C. Beckermann, and I. Steinbach, "Simulation of convection and ripening in a binary alloy mush using the phase-field method," Acta Materialia, vol. 47, no. 13, pp. 3663--3678, 1999.

[12] R. Mendoza, I. Savin, K. Thornton, and P. Voorhees, "Topological complexity and the dynamics of coarsening," Nature Materials, vol. 3, no. 6, pp. 385--388, 2004.

[13] W. Boettinger, J. Warren, C. Beckermann, and A. Karma, "Phase-field simulation of solidification 1," Annual Review of Materials Research, vol. 32, no. 1, pp. 163--194, 2002.

[14] J. A. Warren and W. J. Boettinger, "Prediction of dendritic growth and microsegregation patterns in a binary alloy using the phase-field method," Acta Metallurgica et Materialia, vol. 43, no. 2, pp. 689--703, 1995.

[15] J. A. Warren, R. Kobayashi, A. E. Lobkovsky, and W. Craig Carter, “Extending phase field models of solidification to polycrystalline materials," Acta Materialia, vol. 51, no. 20, pp. 6035--6058, 2003.

[16] S. Wise, J. Lowengrub, J. Kim, K. Thornton, P. Voorhees, and W. Johnson, "Quantum dot formation on a strain-patterned epitaxial thin film," Applied Physics Letters, vol. 87, no. 13, p. 133102, 2005.

[17] Y. U. Wang, Y. M. Jin, and A. G. Khachaturyan, "Phase field microelasticity modeling of surface instability of heteroepitaxial thin films," Acta Materialia, vol. 52, no. 1, pp. 81--92, 2004.

[18] L.-Q. Chen, "Phase-field method of phase transitions/domain structures in ferroelectric thin films: A review," Journal of the American Ceramic Society, vol. 91, no. 6, pp. 1835--1844, 2008.

[19] D. Seol, S. Hu, Z. Liu, L. Chen, S. Kim, and K. Oh, "Phase-field modeling of stressinduced surface instabilities in heteroepitaxial thin films," Journal of Applied Physics, vol. 98, no. 4, p. 044910, 2005. 
[20] C. Shen and Y. Wang, Fundamentals of Modeling for Metals Processing, vol. 22A of ASM Handbook. ASM International, 2009.

[21] L.-Q. Chen, "Phase-field models for microstructure evolution," Annual Review of Materials Research, vol. 32, no. 1, pp. 113--140, 2002.

[22] N. Moelans, B. Blanpain, and P. Wollants, "An introduction to phase-field modeling of microstructure evolution," Calphad, vol. 32, no. 2, pp. 268--294, 2008.

[23] B. Nestler and A. Choudhury, "Phase-field modeling of multi-component systems," Current Opinion in Solid State and Materials Science, vol. 15, no. 3, pp. 93--105, 2011.

[24] I. Steinbach, "Phase-field models in materials science," Modelling and Simulation in Materials Science and Engineering, vol. 17, no. 7, p. 073001, 2009.

[25] Y. Wang, H. Wang, L. Chen, and A. Khachaturyan, "Microstructural development of coherent tetragonal precipitates in magnesium-partially-stabilized zirconia - a computer simulation," Journal of the American Ceramic Society, vol. 78, pp. 657--661, MAR 1995.

[26] Y. Wang, D. Banerjee, C. Su, and A. Khachaturyan, "Field kinetic model and computer simulation of precipitation of $\mathrm{Ll}(2)$ ordered intermetallics from FCC solid solution," Acta Materialia, vol. 46, pp. 2983--3001, MAY 221998.

[27] A. Artemev, Y. Jin, and A. Khachaturyan, "Three-dimensional phase field model of proper martensitic transformation," Acta Materialia, vol. 49, pp. 1165--1177, APR 19 2001.

[28] M. Castro, "Phase-field approach to heterogeneous nucleation," Phys. Rev. B, vol. 67, p. 035412, 2003.

[29] J. Simmons, C. Shen, and Y. Wang, "Phase field modeling of simultaneous nucleation and growth by explicitly incorporating nucleation events," Scripta Materialia, vol. 43, no. 10, pp. 935--942, 2000.

[30] J. Simmons, Y. Wen, C. Shen, and Y. Wang, "Microstructural development involving nucleation and growth phenomena simulated with the Phase Field method," Materials Science and Engineering A-Structural Materials Properties Microstructure and Processing, vol. 365, no. 1-2, pp. 136--143, 2004.

[31] M. Park and R. Arroyave, "Concurrent nucleation, formation and growth of two intermetallic compounds (Cu6Sn5 and Cu3Sn) during the early stages of lead-free soldering," Acta Materialia, vol. 60, no. 3, pp. 923--934, 2012.

[32] S. Hu and L. Chen, "Solute segregation and coherent nucleation and growth near a dislocation - A phase-field model integrating defect and phase microstructures," Acta Materialia, vol. 49, no. 3, pp. 463--472, 2001. 
[33] W. Zhang, Y. Jin, and A. Khachaturyan, "Phase field microelasticity modeling of heterogeneous nucleation and growth in martensitic alloys," Acta Materialia, vol. 55, no. 2, pp. 565--574, 2007.

[34] L. Granasy, T. Borzsonyi, and T. Pusztai, "Nucleation and bulk crystallization in binary phase field theory," Physical Review Letters, vol. 88, no. 20, 2002.

[35] C. Shen, J. Li, and Y. Wang, "Finding critical nucleus in solid-state transformations," Metallurgical and Materials Transactions A-Physical Metallurgy and Materials Science, vol. 39A, no. 5, pp. 976--983, 2008.

[36] L. Zhang, L.-Q. Chen, and Q. Du, "Morphology of critical nuclei in solid-state phase transformations," Physical Review Letters, vol. 98, no. 26, 2007.

[37] L. Zhang, L.-Q. Chen, and Q. Du, “Diffuse-interface description of strain-dominated morphology of critical nuclei in phase transformations," Acta Materialia, vol. 56, no. 14, pp. 3568--3576, 2008.

[38] L. Zhang, L.-Q. Chen, and Q. Du, "Mathematical and numerical aspects of a phasefield approach to critical nuclei morphology in solids," Journal of Scientific Computing, vol. 37, no. 1, pp. 89--102, 2008.

[39] Y. Wen, J. Simmons, C. Shen, C. Woodward, and Y. Wang, "Phase-field modeling of bimodal particle size distributions during continuous cooling," Acta Materialia, vol. 51, no. 4, pp. 1123--1132, 2003.

[40] X. H. Guo, S. Q. Shi, Q. M. Zhang, and X. Q. Ma, "An elastoplastic phase-field model for the evolution of hydride precipitation in zirconium. Part I: Smooth specimen," Journal of Nuclear Materials, vol. 378, pp. 110--119, AUG 152008.

[41] S. M. Allen and J. W. Cahn, "A microscopic theory for antiphase boundary motion and its application to antiphase domain coarsening," Acta Metallurgica, vol. 27, no. 6, pp. 1085--1095, 1979.

[42] R. Balluffi, S. Allen, and W. Carter, Kinetics of Materials. Hoboken, New Jersey: Wiley-Interscience, 2005.

[43] D. R. Gaston, J. W. Peterson, C. J. Permann, D. Andrs, A. E. Slaughter, and J. M. Miller, "Continuous integration for concurrent computational framework and application development," Journal of Open Research Software, vol. 2, no. 1, p. e10, 2014.

[44] D. R. Gaston, C. J. Permann, J. W. Peterson, A. E. Slaughter, a. Andrš, Y. Wang, M. P. Short, D. M. Perez, M. R. Tonks, J. Ortensi, et al., "Physics-based multiscale coupling for full core nuclear reactor simulation," Annals of Nuclear Energy, 2014.

[45] M. R. Tonks, D. Gaston, P. C. Millett, D. Andrs, and P. Talbot, "An object-oriented finite element framework for multiphysics phase field simulations," Computational Materials Science, vol. 51, no. 1, pp. 20--29, 2012. 
[46] L. Zhang, M. R. Tonks, D. Gaston, J. W. Peterson, D. Andrs, P. C. Millett, and B. S. Biner, "A quantitative comparison between $\mathrm{C} 0$ and $\mathrm{C} 1$ elements for solving the Cahn--Hilliard equation," Journal of Computational Physics, vol. 236, pp. 74--80, 2013.

[47] M. Avrami, "Kinetics of phase change I - General theory," Journal of Chemical Physics, vol. 7, pp. 1103--1112, DEC 1939.

[48] M. Avrami, "Kinetics of phase change II - Transformation-time relations for random distribution of nuclei," Journal of Chemical Physics, vol. 8, pp. 212--224, FEB 1940.

[49] J. Christian, The Theory of Transformations in Metals and Alloys, Part I. New York: Pergamon Press, second ed., 1975.

[50] A. Iserles, A First Course in the Numerical Analysis of Differential Equations. Cambridge, UK: Cambridge University Press, first ed., 1996.

[51] D. Gaston, C. Newman, G. Hansen, and D. Lebrun-Grandie, "MOOSE: A parallel computational framework for coupled systems of nonlinear equations," Nuclear Engineering and Design, vol. 239, pp. 1768--1778, OCT 2009.

[52] B. S. Kirk, J. W. Peterson, R. H. Stogner, and G. F. Carey, "libMesh: a C++ library for parallel adaptive mesh refinement/coarsening simulations," Engineering with Computers, vol. 22, no. 3-4, pp. 237--254, 2006.

[53] N. Dupin, I. Ansara, C. Servant, C. Toffolon, C. Lemaignan, and J. Brachet, "A thermodynamic database for zirconium alloys," Journal of Nuclear materials, vol. 275, no. 3, pp. 287--295, 1999.

[54] S. Hu, J. Murray, H. Weiland, Z. Liu, and L. Chen, "Thermodynamic description and growth kinetics of stoichiometric precipitates in the phase-field approach," Calphad, vol. 31, no. 2, pp. 303--312, 2007. 